Recherches en didactique des langues et des cultures

Les cahiers de l'Acedle

17-2 | 2020

Recherches collaboratives en didactique des langues

\title{
Présentation : les recherches collaboratives en didactique des langues, orientations actuelles
}

Véronique Miguel-Addisu et Nathalie Thamin

\section{OpenEdition}

1 Journals

Édition électronique

URL : https://journals.openedition.org/rdlc/7276

DOI : $10.4000 /$ rdlc.7276

ISSN : 1958-5772

Éditeur

ACEDLE

Référence électronique

Véronique Miguel-Addisu et Nathalie Thamin, «Présentation : les recherches collaboratives en didactique des langues, orientations actuelles », Recherches en didactique des langues et des cultures [En ligne], 17-2 | 2020, mis en ligne le 27 avril 2020, consulté le 17 octobre 2022. URL : http:// journals.openedition.org/rdlc/7276 ; DOI : https://doi.org/10.4000/rdlc.7276

Ce document a été généré automatiquement le 17 octobre 2022.

Creative Commons - Attribution - Pas d'Utilisation Commerciale - Pas de Modification 4.0 International - CC BY-NC-ND 4.0

https://creativecommons.org/licenses/by-nc-nd/4.0/ 


\title{
Présentation : les recherches collaboratives en didactique des langues, orientations actuelles
}

\author{
Véronique Miguel-Addisu et Nathalie Thamin
}

A Marie-Odile Maire-Sandoz, inspiratrice de ce numéro.

\section{Présentation : les recherches collaboratives en didactique des langues, orientations actuelles}

1 Les textes réunis ici s'intéressent aux méthodes, aux savoirs et aux enjeux propres aux recherches que l'on nomme «collaboratives» (désormais RC) en didactique des langues. La notion de collaboration induit un travail en commun (collaborare: travailler ensemble), ce qui inclut la coopération (cum operare : faire ensemble). La collaboration peut s'envisager en termes institutionnels et contractuels, mais c'est davantage à la praxis que les contributions s'intéressent ici, en tant qu'activité pouvant transformer les manières de penser et de faire des collaborateurs lorsqu'ils s'engagent à la fois dans une réflexivité conjointe et des actions complémentaires. Nous retiendrons que les dispositifs de recherche collaborative se distinguent par des « processus impliquant chercheurs et praticiens qui collaborent pour élucider une question de recherche, plus ou moins codécidée, afin de produire des savoirs, le plus souvent coénoncés et covalidés» (Bourrassa et Boudjaoui, 2012: 5). Faire de la recherche "avec» les praticiens (plutôt que "pour" ou "sur») pose cependant un certain nombre de questions à éclairer : des objets et méthodes spécifiques émergent-ils en didactique des langues? À quels enjeux scientifiques et sociaux peut répondre ce type de recherche qualitative ? Pour quels effets?

Dans la première partie de cette présentation, nous contextualiserons ces questions en proposant quelques repères conceptuels sur lesquels s'appuient la plupart des 
contributeurs. Ces premiers jalons débouchent sur un premier trait définitoire des textes présentés ici : la spécificité d'une écriture qui, dans sa forme-même, témoigne du travail de co-énonciation et de co-validation des auteurs.

3 La seconde partie de cette présentation introduit les textes selon quatre axes, que nous avons choisis parce qu'ils nous semblent à même de mettre l'accent sur des éléments saillants de la RC en didactique des langues aujourd'hui. Un premier constat nait de la lecture des onze contributions réunies ici : la définition de départ que nous avions proposée dans l'appel à contribution implique que les rôles de chercheur ou de professionnel, de formateur ou de praticien soient clairement identifiés, et identifiables par les collaborateurs. La plupart des contributions que nous réunissons ici montrent cependant que la collaboration appelle surtout une transformation de ces rôles tout au long du processus collaboratif, et que l'importance de la relation entre les acteurs prend le pas sur celle des fonctions professionnelles (axe 1).

4 Ce numéro fait aussi comprendre que si les liens entre formation et recherches collaboratives sont particulièrement forts, ces deux champs ne se confondent pas pour les acteurs, distinction qui est à l'origine du processus à l'œuvre dans les différents projets présentés (axe 2). Cette intuition a été à l'origine de notre appel à contribution, qui a émergé en avril 2018, lors d'un séminaire de formation à l'Institut français d'éducation (IFE) qui réunissait chercheurs et formateurs en vue d'explorer de nouvelles pistes didactiques avec les élèves allophones ${ }^{1}$. Outre la thématique du séminaire, les projets et travaux soumis à la réflexion du groupe avaient tous la particularité d'émaner d'une collaboration pratique entre chercheurs et praticiens: cette praxis apparaissait comme essentielle pour la formation, ainsi que pour la recherche. Le troisième constat que l'on peut faire à la lecture de ce numéro est que la praxis renvoie immanquablement à des positionnements éthiques situés, et contextualisés, qu'il appartient aux chercheurs de clarifier et d'assumer socialement (axe 3). Aujourd'hui, la didactique des langues transpose explicitement ces questions hors de la classe: de jeunes chercheurs explorent ces voies au sein de partenariats innovants dont les premiers résultats sont prometteurs (axe 4).

5 Nous conclurons cette présentation en revenant sur quelques points qui demeurent délicats dans le contexte actuel pour que des recherches collaboratives se déploient entre chercheurs universitaires et institutions partenaires.

\section{Repères méthodologiques, épistémologiques et éthiques des recherches collaboratives pour la didactique des langues}

6 De nombreuses disciplines de recherche ont développé une réflexion épistémologique significative sur les recherches dites collaboratives ou participatives, sans que ces termes fassent consensus sur les enjeux qu'ils recouvrent (Bourdieu, 1993 ; Desgagné et Bednarz, 2005 ; Mesny et Mailhot, 2010 ; Lenoir, 2012 ; Gillet et Tremblay, 2017). La nature hétéroclite du concept se perçoit par la diversité des ancrages auxquels se réfèrent les chercheurs : «chaque auteur ou collectif d'auteurs présente de ce fait sa propre compréhension de la recherche collaborative, empruntant tantôt à la recherche action, à la recherche-intervention, à la recherche participative et aux Lesson Studies " (Morrissette, PagoniAndreani et Pepin, 2017:3). Ces chercheurs cependant mettent aussi l'accent sur l'intérêt de telles démarches pour contribuer à de nouveaux savoirs répondant à des questions sociales. Les RC appréhendent les savoirs comme des 
éléments largement dépendants de processus complexes, qui contribuent eux-mêmes à définir, et à transformer, ces savoirs (Giddens, 1987). Les RC ont donc un positionnement éthique et épistémologique spécifique, qui induit des choix méthodologiques. Elles débouchent sur de nouvelles connaissances, que l'on pourrait peut-être qualifier d'hybrides, de métissées, d'altéritaire, et de dynamiques.

7 Sur un plan méthodologique, la collaboration crée des espaces d'échanges réflexifs qui obligent chacun à expliciter ses propres cadres interprétatifs et à les éprouver au regard de la pertinence d'autres régimes de vérité. Si les savoirs savants et les savoirs pratiques se distinguent du fait des rôles des acteurs, les contacts sont justement un espace de co-construction des significations: «le praticien peut devenir co-chercheur et le chercheur co-praticien» (Canut, Espinosa \& Vertalier, 2013: 75). Sur le plan épistémologique, les savoirs didactiques s'élaborent dans un entre-deux entre pratique et conceptualisation qui éclairent à la fois la structure organisationnelle et les logiques d'action des acteurs/agents (Vinatier et Morrissette, 2015 ; Bourrassa et al., 2017). Sur le plan éthique, les RC adoptent une perspective critique, avec une visée d'émancipation sociale: la réflexivité conjointe veut réduire l'hégémonie des savoirs savants, elle agit sur les rapports de domination entre sciences et pratiques (Freire, 1977 ; Lechopier, $2013: 6$ ), elle renforce le pouvoir d'action sociale des participants ; les $\mathrm{RC}$ contribuent donc à éclairer les conditions d'une éthique de la participation (Vinatier et Morrissette, 2015).

8 Ces démarches ont tendance à se développer en France dans le champ de l'éducation (et en didactique des langues en particulier), mais elles sont encore peu documentées : on relève que la réflexion portant sur les collaborations se retrouve le plus souvent associée aux recherches-actions et à leurs effets sur l'environnement social dans lequel elles s'inscrivent (Astolfi, 1993; Fijalkow et Ragano, 1999; Narcy-Combes, 2001 ; Macaire, 2007 ; Castellotti, 2013). Au Canada, les RC font l'objet d'analyses qui interrogent plutôt la démarche compréhensive (et donc interprétative) construite entre les acteurs selon un principe de double vraisemblance, et d'intersubjectivité (Desgagné et al., 2001 ; Anadón, 2007 ; Bednarz, 2013). C'est à cet aspect que nous nous intéresserons tout particulièrement dans ce dossier, car le partage d'expertise, et l'effacement des frontières entre communauté professionnelle et communauté scientifique qu'induit la RC, nous semble particulièrement heuristique en didactique des langues. Et c'est bien cet aspect des choses que les contributeurs-trices de ce numéro abordent dans leurs textes, notamment dans leurs choix énonciatifs.

\section{Diversité des terrains et des objets pour un processus partagé}

9 Les objets des RC étudiées dans ce numéro portent sur un ensemble de dispositifs didactiques, la plupart se déroulent en contexte scolaire (maternelle, primaire, secondaire), en classe ordinaire ou en UPE2A (Unité Pédagogique pour Elèves Allophones Arrivants), mais concernent également la formation initiale d'étudiants en INSPE. Les types de RC explorés et analysés sont des recherches-formations, des Lesson Studies, des projets artistiques, des partenariats pédagogiques... elles mobilisent une collaboration entre chercheurs et enseignants, conseillers pédagogiques, familles, inspecteurs de l'éducation nationale, étudiants en formation initiale, communautés autochtones (contexte Amérique du nord), directeur de théâtre et artistes en résidence, praticiens de l'éducation muséale (directrice de musée, médiatrices culturelles). 
Dans neuf contributions sont évoquées des recherches contribuant à la formation des enseignants. Sept s'intéressent à des objets "scolairement identifiés " tels que la syntaxe et la ponctuation (Giguère et al.), l'écriture (Kervyn), la recherche documentaire d'enseignants d'anglais du secondaire (Bento), la didactique de l'oral en contexte plurilingue (Miguel Addisu et Beaumont), la lecture d'albums de jeunesse (Deschoux et al.), la formation à la didactique du plurilinguisme (Colombel et Fillol ; Macaire). Deux problématisent surtout les dynamiques de transformation des acteurs (Lemaire et al., Lebreton et Lorilleux). Mais les RC ouvrent la didactique à des lieux autres : dans deux contributions sont aussi évoquées des situations dans lesquelles la didactique du plurilinguisme croise les pratiques muséales et artistiques (Carinhas et al. ; Serusclat-Natale et al.).

11 Les onze contributions s'inscrivent dans différents ancrages de la didactique des langues : didactique du français langue maternelle, $d u$ français langue de scolarisation, didactique de l'anglais, didactique du plurilinguisme en contexte minoritaire ou majoritaire francophone. Elles s'ancrent également dans différents contextes géographiques: Canada anglophone (1 Alberta) et Canada francophone (1 Québec), France métropolitaine (5), territoire ultramarin de Nouvelle Calédonie (1), États-Unis (1), Suisse romande (1), Uruguay (1). Dans l'argumentaire initial du numéro, plusieurs axes avaient été envisagés émanant des questionnements soulevés par les coordinatrices du numéro: 1) Recherches collaboratives et diffusion des savoirs: enjeux. 2) $R C$ et engagement des acteurs: savoirs. 3) RC et processus de co-construction des savoirs: méthode. Force est de constater qu'aucun article ne s'inscrit dans un axe exclusif, mais tous en traitent en partie. S'agit-il de signes de l'émergence d'un paradigme de recherche en didactique des langues? On peut faire l'hypothèse que les RC bousculent les catégorisations consensuelles et mettent à jour ce qui est de l'ordre de l'entre deux : «l'entre n'a rien en propre, ne possède pas de statut, par conséquent passe inaperçu. En même temps, l'entre est par où tout "passe", "se passe ", peut se déployer " (Jullien, 2012: 51).

12 Cette démarche se lit tout d'abord dans les procédés d'énonciation que tous les auteurs ont investis de façon à rendre compte de la collaboration à l'œuvre dans les recherches dont ils font état.

\section{Un processus d'écriture collaborative}

13 En concordance avec la thématique proposée, les coordinatrices avaient fait le pari d'un numéro où l'écriture collaborative serait présente, en invitant explicitement à cette démarche de co-écriture. Le défi a été relevé par plusieurs auteurs, permettant ainsi de mieux rendre compte du processus scientifique qu'impliquent les recherches collaboratives.

Trois textes sont co-signés par des chercheurs et des professionnels (Lemaire, Beauparlant \& Howse ; Miguel Addisu et Beaumont ; Serusclat-Natale et Marzin). Trois textes mêlent les voix de plusieurs chercheurs engagés dans un même projet collaboratif (Giguère, Nadeau, Fisher, Arseneau, \& Quevillon Lacasse ; Carinhas, Araujo \& Moore ; Colombel et Fillol). Les auteures assument une double-voix de praticiennes et chercheures dans deux contributions (Deschoux et Taisson; Lorilleux et Lebreton). Dans les trois textes qui n'ont qu'un seul auteur, l'énonciation joue un rôle premier 
dans l'explicitation des rôles et dynamiques collaboratives qui sont discutées (Bento ; Kervyn ; Macaire).

Ces choix énonciatifs témoignent selon nous de la créativité à l'œuvre dans les RC : les textes écrits à deux voix ou parfois plus, très peu habituels dans les habitus culturels académiques, ont nécessité de la part des auteur-e-s (ainsi que pour les relecteurs!) un certain nombre de réajustements, notamment d'ordre énonciatif, de clarification des processus d'écriture, des places et rôles des co-auteurs dans la recherche. Cela a favorisé dans le processus d'écriture lui-même la dimension réflexive sur le projet et la démarche collaborative. Les auteurs ont exploré des procédés divers comme les notes de bas de page explicatives des choix opérés, le jeu sur les marques typographiques pour distinguer les différents énonciateurs. On trouve ainsi sous les plumes de Maud Serusclat-Natale et Yannick Marzin l'explicitation suivante en note 5 :

« Lorsque nous avons écrit cet article, souvent à distance, notre co-écriture a pris la forme d'un dialogue plus personnel, dont on trouve les traces syntaxiques (pronoms, marques d'adresse, questions en suspens, etc.) dans les extraits qui vont suivre. C'est volontairement que nous laissons apparaitre ce glissement sémantique puisqu'il est une manifestation concrète de la pensée que nous développons dans cette contribution ».

Dans son article écrit à une voix, Bernadette Kervyn précise les choix énonciatifs opérés dans la première note de bas de page pour mieux retracer le processus collaboratif à l'œuvre :

«Malgré l'usage fréquent du pronom «nous» en recherche pour se désigner en tant qu'auteur et parfois induire une intention de neutralité axiologique, certes toute relative, dans le présent article, choix est fait de recourir à une écriture en «je » quand il s'agit de l'auteur. Ce choix permettra de réserver l'usage du «nous » pour désigner le collectif engagé dans la recherche dont il sera question. Seront aussi utilisés de façon distincte le «nous » et le " on », ce dernier visant à inclure les lecteurs potentiel».

Un dernier exemple non exhaustif des positionnements est celui choisi conjointement par Emilie Lebreton et Joanna Lorilleux qui, en jouant sur la typographie, assument deux voix distinctes tout en soutenant le fait que chacune de ces voix est à la fois celle d'une chercheure et d'une praticienne :

« Notre écriture commune ne peut laisser se confondre nos voix, nos regards, nos expériences. C'est pourquoi nous avons fait le choix d'utiliser l'italique, précédé des initiales de l'énonciatrice (E.L. pour Emilie Lebreton et J.L. pour Joanna Lorilleux) pour marquer les passages où le croisement, le frottement de nos expériences fait sens. Les passages sans italiques correspondent à des compréhensions partagées des phénomènes présentés ».

\section{Organisation du numéro}

Nous avons choisi de présenter les contributions selon quatre axes complémentaires: un premier axe traite de la collaboration entre chercheurs et praticiens sous l'angle de la complémentarité qui se distingue cependant du consensus. Un second se centre sur les effets que la collaboration possède sur le développement professionnel des praticiens. Un troisième apporte une réponse éthiquement située de la recherche à des questions socialement vives. Enfin, un quatrième axe présente deux recherches doctorales en cours qui interrogent la posture et l'implication du chercheur dans des partenariats innovants (festival, musée). 
Axe 1 : La collaboration entre chercheurs et praticiens : une complémentarité qui diffère du consensus

19 La première contribution présente une expérience de mise en œuvre de recherche collaborative entre une enseignante chercheure de Paris Descartes Margaret Bento, et cinq enseignantes d'anglais dans le secondaire en France. La recherche s'inscrit dans le cadre d'une étude portant sur l'usage des ressources pédagogiques et du travail documentaire (projet ANR ReVEA, Ressources vivantes pour l'enseignement et l'apprentissage), sur la manière de didactiser, d'organiser le contenu des séquences pédagogiques, sur l'éthique professionnelle que ces choix engendrent ou qui est engendrée par ces choix. L'analyse met en évidence une différence de logique ainsi qu'une complémentarité de la part des enseignantes et de la chercheure : pour les unes la collaboration a pour finalité le développement professionnel, et pour l'autre la production de connaissances. L'auteure met en avant le fait que la double fonction de recherche et de formation dans une approche collaborative (faire de la recherche avec) ne va pas de soi, et peut "produire une forme de malaise au travail dans le questionnement perpétuel qu'il enclenche chez les praticiennes ».

20 Enseignante chercheure à l'INSPE de Bordeaux, Bernadette Kervyn s'interroge sur les caractéristiques essentielles des recherches de type collaboratif qui contribuent à produire des ressources de formation de qualité. Dans le champ de la didactique du français, la contribution prend comme corpus le processus d'ingénierie didactique engagé suite à la recherche pluridisciplinaire LireÉcrireCP (Goigoux et al., 2016) dans l'objectif de produire des outils sur l'enseignement de l'écriture au début de l'école élémentaire. L'auteure situe son propos au cœur des préoccupations praxéologiques de la didactique en tant que discipline productrice de connaissances et de ressources pour l'action. La RC engagée sur ce volet écriture a été menée avec une équipe de formateurs exerçant en Aquitaine ainsi qu'avec le Centre Alain Savary (CAS) de l'Institut Français de l'Éducation (IFÉ). Ce projet a consisté en la production de quatre scénarios de formation sur l'enseignement de l'écriture au CP ainsi que de ressources d'interface plus générales sur le processus scriptural et son enseignement, et sur les résultats de la recherche LireÉcrireCP en matière d'écriture. Dans sa contribution, l'auteure examine la complémentarité des logiques des chercheurs et des praticiens qui rend la recherche utile en didactique, et donc nécessaire.

21 Véronique Miguel Addisu, enseignante chercheure à l'INSPE de Rouen, et Sophie Beaumont, inspectrice de l'Éducation nationale dans le réseau AEFE (Agence pour l'Enseignement Français à l'Étranger) analysent à deux voix le processus de collaboration $\mathrm{du}$ projet dont elles sont co-pilotes, les transformations et les déplacements professionnels initiés ainsi que les contraintes induites. École, Parole de l'élève et Plurilinguisme: Recherche Collaborative au lycée français de San Francisco - AEFE, USA (E2PRC_Francisco) prend pour objet la didactique de l'oral en contexte plurilingue et réunit un ensemble d'acteurs (enseignants, formatrices, chercheurs, inspectrice). Les auteures montrent comment le recueil de données sociolinguistiques réalisé par les chercheurs du projet a contribué à une démarche partagée d'«apprenance ", par laquelle tous les acteurs ont transformé leurs gestes professionnels en alliant pratique, recherche et développement professionnel.

Axe 2: Les effets de la collaboration sur le développement professionnel des praticiens 
22 la formation à et par la recherche des futurs enseignants dans le cadre de la rénovation des INSPE se pose actuellement de manière accrue. La réalité des classes françaises multiculturelles et multilingues nécessite de rendre lisibles les travaux inscrits dans le paradigme du plurilinguisme, dans une perspective de "recherche-formation » qui se caractérise par sa dimension collaborative à double sens, de et vers la formation, de et vers la recherche. L'auteure analyse les conditions d'élaboration de cette collaboration au regard des enjeux formatifs et de recherche, articulés les uns avec les autres autour de notions comme celle de plurilinguisme. Pour l'auteure, la recherche soutient le développement professionnel, y compris en formation initiale ; la démarche propre à la « recherche-formation » nécessite d'être davantage exploitée en formation initiale.

Carole-Anne Deschoux et Claire Taisson rendent compte d'une recherche collaborative outillée d'un dispositif de formation de Lesson Study portant sur l'usage d'albums de littérature jeunesse à l'école primaire. L'objectif de cette RC vise à développer des pratiques orientées non plus exclusivement vers le code ou la lecture oralisée, mais aussi vers la compréhension de ces matériaux souvent composites, la planification et la mise en tâches de l'enseignement en littérature. Les auteures cherchent à montrer le principe dynamique construit par la collaboration d'enseignantes volontaires et de chercheures praticiennes ${ }^{2}$ dans le cadre d'une formation continue en didactique du français dispensée dans un institut de formation en Suisse romande. La contribution montre que chercheurs et praticiens font ensemble, mais que cela induit des effets différents et complémentaires qui contribuent largement au développement professionnel des praticiens : la pratique partagée, et les analyses qui en découlent, sont un levier puissant de transformation en vue de s'adapter à la diversité des démarches d'apprentissage des élèves. Rosianne Arseneau, et Claude Quevillon Lacasse, porte sur l'accompagnement professionnel de praticiens dans le développement de dispositifs didactiques en syntaxe et en ponctuation pour améliorer les compétences en écriture d'élèves de 10 à 14 ans. Dans cette contribution, les analyses se focalisent sur le regard de conseillères pédagogiques impliquées dans le projet. L'équipe de recherche était composée de chercheures, de conseillères pédagogiques et d'enseignants du primaire et du secondaire au Québec. Le point de vue des conseillères pédagogiques montre que la recherche collaborative permet de documenter l'efficacité d'un triple accompagnement (rencontres collectives mensuelles, observations en classe avec rétroaction personnelle, matériel offert) et de partager des rôles traditionnellement plus cloisonnés.

Axe 3: Une réponse éthiquement située de la recherche à des questions socialement vives

Eva Lemaire, René Beauparlant et Cécile Howse présentent un projet de rechercheaction-formation (RAF) en éducation, ancré dans le contexte canadien albertain. Il implique le terrain scolaire (élémentaire) et une collaboration avec la communauté autochtone, dans une optique de réconciliation des peuples autochtones et de revitalisation d'une langue en voie de disparition, la langue michif. Le postulat de la recherche était que « le michif pourrait constituer un pont entre les Métis et les élèves des écoles francophones et d'immersion française dont la langue d'étude est le français, dans un contexte par ailleurs anglophone majoritaire ». L'approche dite de l'éveil aux langues et l'apport des pédagogies autochtones ont guidé la création de matériel 
pédagogique. Inspirée des recherches participatives et d'une méthodologie de recherche autochtone nommée "visiting way methodology» (Gaudet, 2019), Eva Lemaire interroge tout particulièrement l'engagement, la posture, la question de la responsabilité et la légitimité du chercheur dans ce contexte de réconciliation où l'implication des partenaires autochtones est décisive " pour soutenir la légitimité de la recherche et maintenir des relations éthiques entre monde académique et communautés autochtones ». Les auteurs insistent sur la nécessité de développer une "éthique de la relation" avec tous les partenaires de la recherche, en particulier autochtones, et de revaloriser les savoirs autochtones. Ils en montrent l'intérêt scientifique. Par ailleurs, la RAF a favorisé l'expertise des praticiens et l'articulation entre les savoirs de la chercheure et les savoirs de l'enseignant.

L'une des finalités majeures des recherches en sociodidactique de Claire ColombelTeuira et de Véronique Fillol, enseignantes-chercheures en Nouvelle Calédonie, est de contribuer au développement d'une perspective plurilinguistique pour une école plus inclusive des pratiques langagières des élèves, à la décolonisation des savoirs concernant les dynamiques plurilingues en contexte scolaire, et plus encore à l'émancipation des outils de l'école. C'est dans cet esprit qu'elles proposent, avec la contribution de Vahimiti Bousquet, professeure des écoles, une analyse critique et réflexive de deux programmes de recherche qu'elles ont menés, École Plurilingue Outremer ЕсОLРОм, 2009-2011 et Je lis j'écris le monde (2017-2018), concernant l'inclusion des langues océaniennes dans le contexte diglossique calédoniens. Les auteures analysent les processus et les implications de la co-élaboration des savoirs dans cette situation postcoloniale spécifique et s'interrogent sur les conditions de réussite d'une activité de recherche qui, comme elles le rappellent, constitue toujours un acte d'exercice de pouvoir, en ce qu' "elle construit des réalités sociales et les légitime à travers sa vulgarisation parfois sans l'accord des individus». La comparaison des deux projets soulève des questions épistémologiques et éthiques et c'est la recherche-actionintervention du second projet qui parait aux auteures la plus efficiente sur les plans éthique, scientifique, et social.

S'appuyant sur leurs expériences de recherche respectives et par une mise en discussion réflexive, les enseignantes chercheures Emilie Lebreton et Joanna Lorilleux explorent les dimensions épistémologiques, éthiques et relationnelles qui déterminent leur engagement dans ces démarches collaboratives. Elles interrogent les catégories mobilisées habituellement et envisagent sous l'angle expérientiel la manière dont les savoirs se construisent. Elles mettent au second plan la notion de "savoirs" (savants ou pratiques) au bénéfice de la relation qui, lorsqu'elle se construit par la collaboration, renvoie selon elles à la fois à l'impossibilité du consensus, et à la fécondité scientifique de la subjectivité en actes. Autrement dit, les dynamiques collaboratives transforment toutes les pratiques, ce qui pour les auteures fait considérer les RC comme relevant d'un paradigme herméneutique.

Axe 4 : Deux recherches doctorales en cours : posture, implication du chercheur et exploration de partenariats originaux

Maud Serusclat-Natale, doctorante en sciences du langage (Université Paul Valéry, Montpellier, sous contrat CIFRE avec MA scène nationale (structure publique) du Pays de Montbéliard) et Yannick Marzin, directeur de cette même scène, composent un texte écrit à quatre mains portant sur un projet partenarial entre MA et l'éducation nationale, nommé Parlemonde. Ce projet, par ailleurs objet de recherche, a pour 
ambition d'embarquer artistes, élèves EANA, enseignants et chercheur, dans "une démarche de création et de production d'œuvres artistiques plurielles et plurilingues ». Les auteurs posent la question de la place du chercheur dans la collaboration, sa posture et sa légitimité symbolique. Y sont développées la notion centrale de "chercheur embarqué » ainsi qu'une réflexion sur la posture de salarié du doctorant Cifre, engagé dans un cadre de recherche singulier et paradoxal qui nécessite une articulation entre «les nécessités d'une pratique opérationnelle et les exigences d'une recherche ancrée dans le paradigme de la «low science ", de l'« épistémologie de la relation », et d'une co-production collective.

Raquel Carihnas, Marie Helena Araújo e Sá et Danièle Moore, explorent un projet partenarial entre musées, familles et école en Uruguay. Elles montrent qu'il existe peu de recherches encore pour comprendre comment les musées peuvent contribuer à une didactique plurilingue altéritaire. Elles montrent, à l'instar du texte précédent, que la doctorante a un rôle essentiel de médiation entre les acteurs, rôle parfois difficile à endosser. L'expérience analysée ici concerne les débuts du projet, et fait comprendre combien le partenariat institutionnel bouscule les habitudes des participants : il n'est possible que parce que chaque partenaire agit en assumant un pouvoir qui lui est propre, en vue d'initier une relation avec tous. Dans l'univers muséal encore mal connu du champ de la didactique du plurilinguisme, l'implication concrète du chercheur permet d'identifier les difficultés, ainsi que les leviers de ce type de partenariat encore mal connu.

\section{En guise de discussion}

30 Ce numéro permet d'avoir une idée des domaines dans lesquels se développent aujourd'hui des recherches collaboratives en didactique des langues. Les RC problématisées ici s'inscrivent toutes résolument dans un paradigme qualitatif. S'intéressant aux praxis et à leurs effets sur les milieux de pratiques, les contributions convergent au moins sur deux points : elles demeurent marginales, et participent à la fois au développement professionnel des acteurs et à l'émergence de nouveaux savoirs scientifiques. Il nous semble que ces recherches disent quelque chose des limites atteintes par un modèle descendant de transposition recherche/formation qui s'appuie sur la différence et les frontières entre ces deux lieux de référence, plutôt que sur la circulation des savoirs. On comprend ici qu'il ne s'agit pas d'opposer recherche et formation en didactique des langues: ces études permettent de documenter et de poursuivre l'effort de conceptualisation de leur relation. En réaménageant les pouvoirs entre savoirs pratiques et savoirs savants, c'est une démarche critique particulière qui s'opère dans les RC pour tous les acteurs, celle de la capabilité.

31 Pourtant les appels de plus en plus nombreux à des collaborations de principe entre chercheurs et praticiens invitent aussi à une réflexion politique dont la didactique des langues ne peut s'affranchir : à les développer avec le soutien des décideurs, ne courton pas le risque d'instrumentaliser les RC au bénéfice d'une culture de la compétitivité qui impose à la fois collaboration et résultats probants des formations s'y inscrivant ? Nous ne pouvons non plus ignorer les projets de recherches collaboratives qui n'ont pas abouti, et pour lesquels nous n'avons pas reçu de proposition. Les chercheurs ayant contribué s'interrogent sur leur rôle, et se positionnent face à l'institution partenaire, ils montrent tous que dans les projets auxquels ils ont participé, leur engagement est 
considéré comme possible, souhaitable, et bénéfique. Mais toutes les voix réunies dans ce numéro soulignent aussi ô combien est délicate à mettre en place une dynamique de recherche collaborative qui contribue pleinement à la formation. Cette préoccupation est loin d'être nouvelle dans le champ des didactiques :

«Au chercheur qui accepte de répondre à une demande qui, dans la majorité des cas, est institutionnelle, c'est-à-dire produite par un responsable administratif (IEN en général), la question se pose de savoir en quoi sa réponse est spécifique, c'est-àdire différente à la fois de celle du formateur qu'il pourrait rencontrer sur ce même terrain, et surtout en quoi elle peut être reconnue comme recherche par la communauté scientifique à laquelle il appartient. C'est ce second problème qui est le plus épineux, à telle enseigne qu'il semble que, faute de trouver de réponse satisfaisante à celui-ci, nombre de chercheurs préfèrent décliner l'offre qui leur est faite. » (Fijalkow et Ragano, 1999 : 19)

On découvrira dans ce numéro des contributions qui montrent que les RC déploient des processus scientifiquement rigoureux, qui favorisent des méthodes de recherche altéritaires, certes inconfortables, mais fécondes en didactique des langues. En favorisant de nouvelles connaissances fondées sur la relation, l'écart, et la capabilité, ces démarches contribuent notamment au développement professionnel de tous les acteurs « embarqués», dont les chercheurs.

\section{BIBLIOGRAPHIE}

Anadón, M. (dir) (2007). La recherche participative : multiples regards. Québec : Presses de l'Université du Québec.

Astolfi, J.-P. (1993). « Trois paradigmes pour les recherches en didactique », Revue française de pédagogie, 103, 5-18.

Bourdieu, P. (dir.) (1993). La misère du monde. Éditions du Seuil : Points.

Bourrassa, B. \& Boudjaoui, M. (2012). Des recherches collaboratives en sciences humaines et sociales (SHS) : Enjeux, modalités et limites. Laval : PUL (Presses de l'Université Laval).

Bourrassa, B., Picard, F., Le Bossé, Y. \&, Fournier, G. (2017). « Accompagner les groupes de recherche collaborative : en quoi consiste ce « faire avec »? », Phronesis 2017/1 (Vol. 6), 60-73. https://www.erudit.org/fr/revues/phro/2017-v6-n1-2-phro03097/1040218ar/.

Canut, E., Espinosa, N. \& Vertalier, M. (2013). « Corpus et prise de conscience des processus interactionnels d'apprentissage du langage pour repenser les pratiques enseignantes en maternelle », Linx, 68-69, https://journals.openedition.org/linx/1489.

Castellotti, V. (2013). « L'articulation recherche-intervention en DDL : comment (ne pas) en sortir? ». In Beacco, J.C. (dir.), Éthique et politique en didactique des langues. Autour de la notion de responsabilité. Paris : Didier. Paris, 74-98.

Desgagné, S. \& Bednarz, N. (2005). « Médiation entre recherche et pratique en éducation : faire de la recherche « avec » plutôt que « sur » les praticiens ». Revue des sciences de l'éducation, 31(2), 245-258. 
Desgagné, S., Bednarz, N., Couture, C., Poirier, L. \& Lebuis, P. (2001). « L'approche collaborative de recherche en éducation : un rapport nouveau à établir entre recherche et formation ». Revue des sciences de l'éducation, 27/1, 33-64.

Fijalkow, J., Ragano, S. (1999). « Recherche-action et entrée dans la culture écrite ». Repères, Recherches-actions et didactique du français. Hommage à Hélène Romian, n²0, 15-28.

Freire, P. (1977). Pédagogie des opprimés. Paris : F. Maspero (traduction française).

Gaudet, C. (2019). Keeoukaywin: The Visiting Way - Fostering an Indigenous Research Methodology. Aboriginal Policy Studies, 7(2), 47-64.

Giddens, A. (1987), La constitution de la société. Paris : PUF.

Gillet, A., Tremblay, D.G. (dir.) (2017). Les recherches partenariales et collaboratives. Québec : Presses Universitaires du Québec.

Goigoux, R. (dir.) (2016). Étude de l'influence des pratiques d'enseignement de la lecture et de l'écriture sur la qualité des apprentissages au cours préparatoire. Rapport de recherche. Lyon, Institut français d'Éducation. En ligne : http://ife.ens-lyon.fr/ife/recherche/lire-ecrire/rapport.

Jullien, F. (2012). L'écart et l'entre. Leçon inaugurale de la Chaire sur l'altérité. Paris : Galilée.

Lechopier, N. (2013). «Recherches participatives et partage de résultats scientifiques. Quels enjeux? ». Bulletin Recherche de l'Institut Français de l'Education, 20, 5-7.

Lenoir, Y. (2012). « La recherche collaborative entre recherche-action et recherche partenariale : spécificités et implications pour la recherche en éducation ». Travail et apprentissages, 9, 14-40.

Macaire, D. (2007). « Notions en Questions : Méthodologies de recherche en didactique des langues ». Recherches en Didactique des langues et des cultures : Les Cahiers de l'Acedle, $n^{\circ} 4$. http:// acedle.org/old/IMG/pdf/Macaire-D_cah4.pdf.

Mesny, A. \& Mailhot, C. (2010). «La collaboration entre chercheurs et praticiens en gestion : Entre faux-semblants et nécessité épistémique ». Revue française de gestion, 202 (3), 33-45.

Morrissette, J., Pagoni-Andreani, M. \& Pépin, M. (2017). « Observation et mise en mots des pratiques dans le cadre des recherches collaboratives : quelles finalités? Quels dispositifs ? Quels impacts?». Phronesis, 6(1-2).

Narcy-Combes, J.-P. (2001) « La recherche-action en didactique des langues : apprentissage, compagnonnage ou évolution libre? », Recherche et pratiques pédagogiques en langues de spécialité, Vol. XXI N², 40-52.

Vinatier, I. \& Morrissette, J. (2015). «Les recherches collaboratives : enjeux et perspectives ». Carrefours de l'éducation, 39(1), 137-170. https://www.cairn.info/revue-carrefours-de-leducation-2015-1-page-137.htm.

\section{NOTES}

1. http://ife.ens-lyon.fr/formation-formateurs/catalogue-des-formations/formation-2017-18/ quelle-didactique-pour-les-eleves-allophones?set_language=en.

2. La formation a rassemblé uniquement des femmes. C'est donc le féminin qui est adopté. 


\section{AUTEURS}

\section{VÉRONIQUE MIGUEL-ADDISU}

INSPE de l'académie de Rouen-Université de Rouen-Normandie, DYLIS EA7474

Mes travaux se situent entre sociolinguistique et didactique, et visent à contribuer à une meilleure connaissance des pratiques langagières des locuteurs plurilingues en situation de contact de langues. À l'école, je cherche à comprendre comment les élèves plurilingues s'approprient la langue de scolarisation en situation éducative immersive en France et à l'étranger, et à construire une approche plurielle de la didactique du français, à visée inclusive, altéritaire.

veronique.migueladdisu[at]univ-rouen.fr

\section{NATHALIE THAMIN}

Université Bourgogne Franche-Comté, CRIT EA3224, Besançon Je suis enseignante-chercheure en sciences du langage à l'Université de Franche-Comté, au sein du Centre de recherches interdisciplinaires et transculturelles (CRIT, EA 3224), et chercheure associée au LIDILEM, Université Grenoble Alpes. Mes recherches actuelles, inscrites en sociolinguistique et didactique des langues, portent sur la scolarisation et la socialisation langagière plurilingues d'élèves dans le contexte de l'école maternelle ainsi que sur les familles en situation de migration ou de mobilité transnationale.

nathalie.thamin[at]univ-fcomte.fr 